\title{
Prognostic Significance of SPARC Expression in Breast Cancer: A Meta-Analysis and Bioinformatics Analysis
}

\author{
Shuai Shi, Hong-Yan Ma, Xin-Ying Han, Yin-Zhou Sang, Ming-Yue Yang, \\ and Zhi-Gang Zhang
}

Department of Pathology, Cangzhou People's Hospital, Cangzhou 061000, China

Correspondence should be addressed to Zhi-Gang Zhang; zhzhg001518@126.com

Received 24 September 2021; Revised 4 December 2021; Accepted 22 January 2022; Published 15 February 2022

Academic Editor: Aziz ur Rehman Aziz

Copyright (c) 2022 Shuai Shi et al. This is an open access article distributed under the Creative Commons Attribution License, which permits unrestricted use, distribution, and reproduction in any medium, provided the original work is properly cited.

\begin{abstract}
Secreted protein, acidic and rich in cysteine (SPARC, also known as osteonectin), is a small molecule glycoprotein associated with cell secretions. The purpose of our research is to clarify the clinicopathological and prognostic significance of SPARC expression in breast cancer. In this study, we performed a meta-analysis and bioinformatics analysis using the PubMed, Web of Science, Wanfang Data, and CNKI databases. The meta-analysis showed that SPARC expression was elevated in breast cancer tissue, compared with normal tissue, while SPARC expression in tumor stromal cells was higher than that of tumor cells. The expression of SPARC was positively correlated with histological grade and TNM staging. The Kaplan-Meier plotter showed that low SPARC expression was negatively correlated with the overall, postprogression, and distant metastasis survival rates of patients. According to Oncomine database, SPARC expression was upregulated in breast cancer than normal tissues. In TCGA database, univariate analysis showed that lymph node metastasis, distant metastasis, and TNM staging were negatively correlated with patient prognosis in breast cancers. Cox multivariate analysis showed that age, lymph node metastasis, distant metastasis, and TNM staging were important factors affecting the survival time of breast cancer patients. SPARC expression can be employed as a good indicator of prognosis of breast cancer patients, which will provide new methods and ideas of preventive treatment.
\end{abstract}

\section{Introduction}

Secreted protein, acidic and rich in cysteine (SPARC, also known as osteonectin), is a small molecule glycoprotein associated with cell secretions [1]. The SPARC gene is located on human chromosome Sq31.3-q32 and contains 10 exons, while the protein contains 298-304 amino acids. SPARC contains three highly conserved domains: the amino-terminal acidic calcium ion domain, the copper ion region homologous to the follicular dormancy hormone, and the extracellular calcium ion region $[2,3]$. Osteopontin (SPARC) is involved in embryonic development, tissue repair, and cell regeneration. SPARC can regulate cell adhesion and cell proliferation through different signaling pathways and is a highly conserved extracellular interstitial protein. Its main functions are to prevent cell adhesion, regulate cell differentiation, prevent the spreading of cells, inhibit cell response to specific growth factors, regulate extracellular matrix and matrix metalloproteinase production, and influence neovascularization $[4,5]$. Many of the biological functions of SPARC are stimulated by interactions with other proteins. Interactions with different proteins can produce a range of functional activities leading to diverse SPARC functions [6]. For example, SPARC participates in the regulation of the extracellular matrix. Its interaction with collagen affects the remodeling of connective tissue.

Additionally, glycoprotein and hyaluronectin contain the same expression site as SPARC, but the two proteins produce opposite effects on cell adhesion and are also associated with tissue remodeling [7]. SPARC can directly bind to vascular endothelial growth factor (VEGF) to inhibit the vascular endothelial growth factor pathway, preventing vascular endothelial growth factor and its receptor from binding to each other [8]. At the same time, SPARC can also bind to platelet-derived growth factor PDGF to indirectly inhibit angiogenesis by downregulating matrix metalloproteinases (MMPs) and 
TABle 1: Main characteristics of eligible studies.

\begin{tabular}{|c|c|c|c|c|c|c|c|c|c|c|}
\hline First author & Year & Country & Ethnicity & Antibody supplier & Cases & Ctr & Risk to cancer & Outcome & Quality & Reference \\
\hline Cui K & 2017 & China & & & 65 & 72 & Increased & & 8 & 24 \\
\hline $\mathrm{Xu} \mathrm{XD}$ & 2014 & China & UK & Abcam & 255 & & & Negative & 7 & 25 \\
\hline Zhou QF & 2020 & China & UK & Abcam & 150 & & & & 7 & 26 \\
\hline Chen F & 2017 & China & USA & Cell Signaling & 122 & & & Negative & 7 & 27 \\
\hline Yan C & 2016 & China & & & 63 & 31 & Increased & & 8 & 28 \\
\hline Chen Y & 2018 & China & China & Bioss & 70 & 20 & Increased & Negative & 8 & 29 \\
\hline Gao LL & 2015 & China & China & Bioss & 60 & 15 & Increased & & 8 & 30 \\
\hline Zhang XM & 2008 & China & USA & Santa & 61 & 32 & Increased & & 8 & 31 \\
\hline Lindner JL & 2014 & German & UK & Novocastra & 667 & & & & 8 & 32 \\
\hline Ma JJ & 2017 & China & USA & Cell Signaling & & & & & 7 & 33 \\
\hline Zhu AJ & 2016 & China & USA & Thermo & 211 & & & & 7 & 34 \\
\hline Guo W & 2017 & China & UK & Abcam & 88 & & & & 8 & 35 \\
\hline Witkiewicz AK & 2010 & USA & Denmark & Dako & & & & & 7 & 36 \\
\hline Watkins G & 2005 & UK & USA & Santa & & & & Negative & 7 & 37 \\
\hline Barth PJ & 2005 & German & UK & Novocastra & 25 & 25 & Increased & & 8 & 38 \\
\hline Sun XY & 2015 & China & China & Bioss & 70 & 20 & Increased & & 8 & 39 \\
\hline Liu XM & 2012 & China & China & Bioss & 65 & 20 & Increased & & 8 & 40 \\
\hline Zhang SQ & 2016 & China & China & Bioss & 92 & 30 & Increased & Negative & 8 & 41 \\
\hline Sun XY & 2014 & China & China & Bioss & 70 & 20 & Increased & & 8 & 42 \\
\hline
\end{tabular}

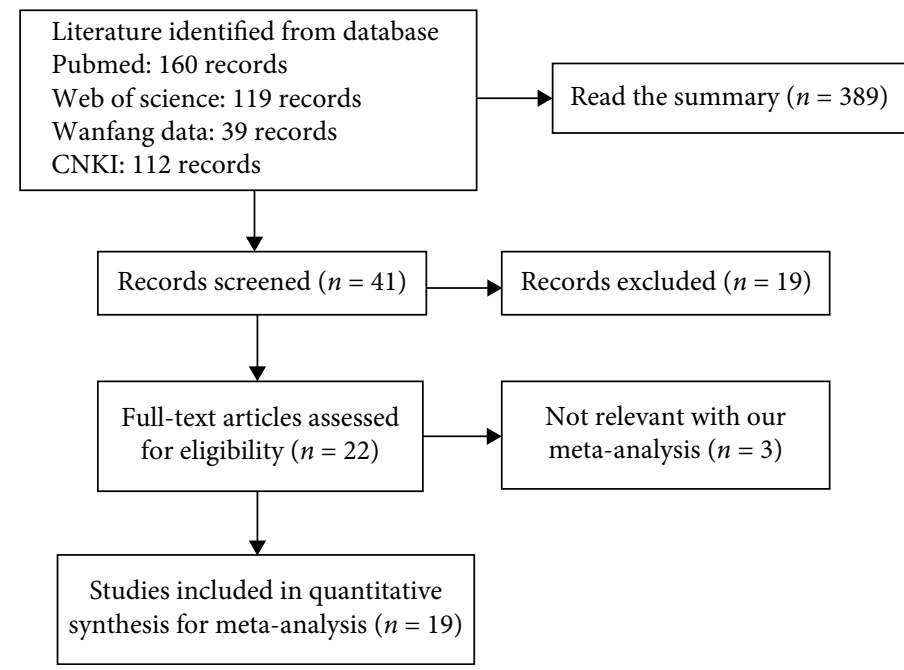

FIgURE 1: Flow diagram of article selection.

transforming growth factor $\beta 1$ antibodies (TGF- $\beta 1$ ), which in turn inhibits tumor invasion and metastasis $[9,10]$. Abnormal methylation of the $\mathrm{CpG}$ island of the promoter region of the SPARC gene can inhibit SPARC expression [11].

Abnormal methylation of the SPARC gene promoter region that leads to gene expression silencing has been observed in primary pancreatic cancer. SPARC mRNA is expressed in nonneoplastic pancreatic ductal epithelial cells but is not found in pancreatic cancer cell lines, which indirectly indicates that the silencing of the SPARC gene can lead to the development of pancreatic cancer $[12,13]$. These findings suggest that the abnormal methylation of the CpG 2 region may be a suitable marker that can be used for the preliminary screening of early pancreatic cancer. SPARC increases the level of phosphorylation of AKT in gliomas through the PI3K/AKT pathway, significantly inhibiting EGF activity in ovarian cancer [14], and can activate GSK3 $\beta$ targets in adipocytes [15]. In mesangial cells, SPARC can enhance the activity of TGF- $\beta$, which leads to an increase in JNK activation and c-Jun phosphorylation [16]. In the Wnt pathway, SPARC can activate ILK, thereby phosphorylating its downstream in GSK-3B target. Phosphorylation of this target can lead to $\beta$-catenin aggregation and ultimately TCF4 activation. This pathway can also inhibit the expression of laminin in melanocytes $[17,18]$.

Previous research has shown that SPARC is closely associated with tumor development and plays a vital role in tumor invasion and metastasis [19]. SPARC expression is 
TABLE 2: Results of meta-analysis of the correlation between SPARC expression and clinical pathological features of breast cancer.

\begin{tabular}{lcccc}
\hline Clinicopathological features & \multicolumn{2}{c}{ Heterogeneity } & \multicolumn{2}{c}{ Test for overall effect } \\
& $I^{2}(\%)$ & $P$ value & Odds ratio $(95 \%$ CI $)$ & $0.66(0.43-1.00)$ \\
Histological grade & 45 & 0.08 & $0.47(0.31-0.71)$ & $<5^{*}$ \\
TNM staging & 61 & $<0.01$ & $0.52(0.25-1.05)$ & $0.01^{*}$ \\
Lymph node metastasis & 85 & $<0.01$ & $1.03(0.76-1.40)$ & $0.86(0.67-1.12)$ \\
Menopausal status & 0 & 0.49 & $0.98(0.59-1.63)$ & 0.87 \\
Size & 0 & 0.92 & $0.78(0.60-1.02)$ & 0.94 \\
ER & 67 & $<0.01$ & $1.08(0.84-1.37)$ \\
PR & 27 & 0.17 & $1.27(0.86-1.89)$ \\
HER2 & 0 & 0.45 & $8.74(4.11-18.58)$ \\
Overall survival & 63 & 0.05 & $0.18(0.10-0.33)$ \\
SPARC tumor cell & 74 & $<0.01$ & 0.07 \\
SPARC stromal cell & 65 & 0.02 & 0.23 \\
\hline
\end{tabular}

TNM: tumor node metastasis; ER: estrogen receptor; PR: progesterone receptor; HER2: human epidermal growth factor receptor-2.

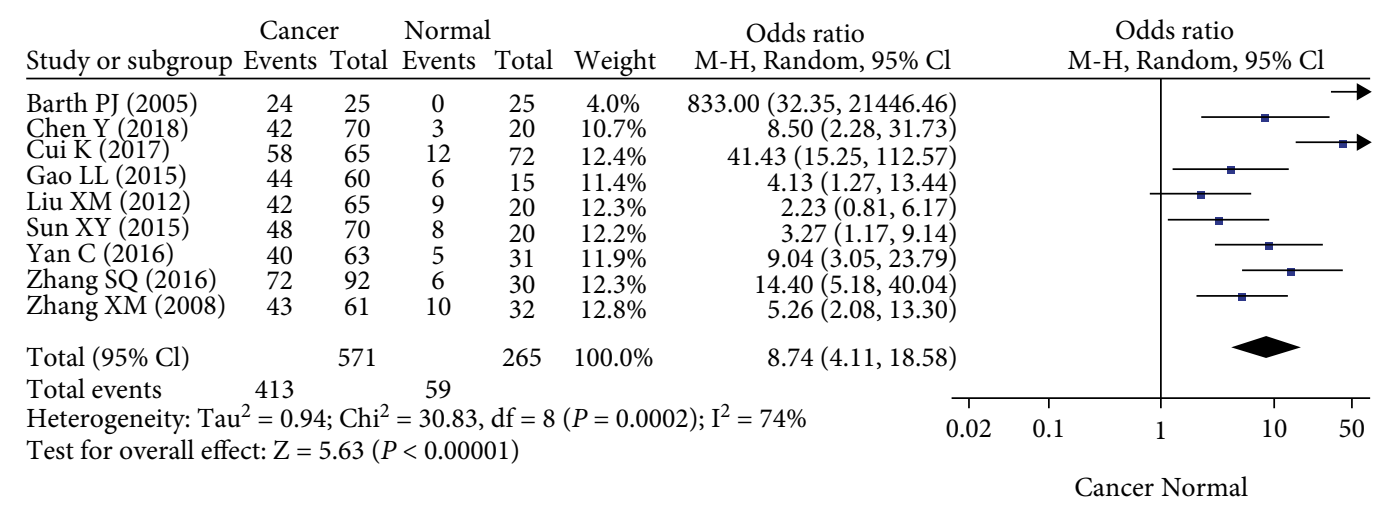

FIGURE 2: Forest plot of the expression of SPARC in breast cancer. Plots of the association between cancer and normal mucosa.

elevated in melanoma, glioma, meningioma, kidney cancer, and prostate cancer [20-23]. High level of SPARC expression enhanced tumor invasion and metastasis, leading to a poor prognosis of patients. In our study, we performed a meta-analysis and bioinformatics analysis to confirm the relationship between SPARC mRNA expression and the clinicopathological factors of breast cancer.

\section{Materials and Methods}

2.1. Static Search and Data Extraction. Articles were searched in PubMed, Web of Science, Wanfang Data, and CNKI databases (May 2020) using the key words: SPARC and breast and cancer or carcinoma or tumor. Inclusion criteria for studies included (1) breast cancer patients; (2) expression of SPARC was detected by immunohistochemistry; (3) articles contain SPARC expression and clinicopathological parameters; (4) all patients did not receive chemotherapy or radiotherapy before surgery. Exclusion criteria included (1) abstracts, case reports, reviews, and meeting notes; (2) repeat publications; (3) unclear diagnosis.

2.2. Data Extraction and Quality Assessment. As shown in Table 1, the information of eligible publications was extracted by two reviewers (Shi S and Zhang ZG) and included name of the first author, year of publication, patients' country, antibody company, number of cases and controls, risks for cancer, and follow-up outcomes. According to the Newcastle Ottawa Oncomine Scale (NOS; ohri.ca/programs/clinical_epidemiology/oxford.htm), the quality of the studies was independently assessed by two reviewers. The methods consists sample selection, comparability, and ascertainment of outcomes.

2.3. Bioinformatics Analysis. The prognostic value of SPARC mRNA expression in breast cancer was analyzed using a Kaplan-Meier plotter (http://www.kmplot.com). Expression of SPARC was associated with overall survival (OS), relapse-free survival (RFS), distant metastasis-free survival (DMFS), and postprogression survival (PPS) for all patients. The expression of SPARC was associated with clinicopathological features. SPARC gene expression was analyzed using the Oncomine database (http://www.oncomine.org), an extensive database of tumor chip data, including gene chip and gene expression data. The database can be used to analyze gene expression differences and classify the clinical information of tumor patients. Differences in SPARC expression at mRNA level were compared between 


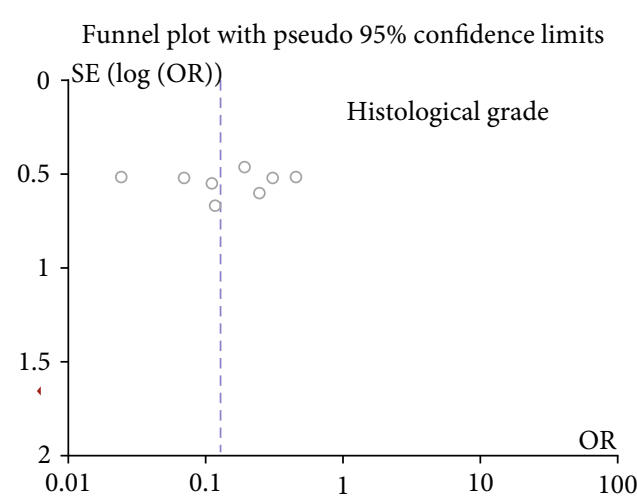

(a)

Funnel plot with pseudo 95\% confidence limits

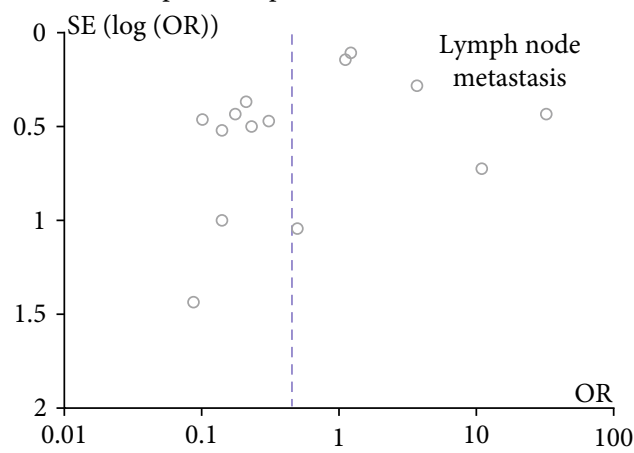

(c)

Funnel plot with pseudo $95 \%$ confidence limits

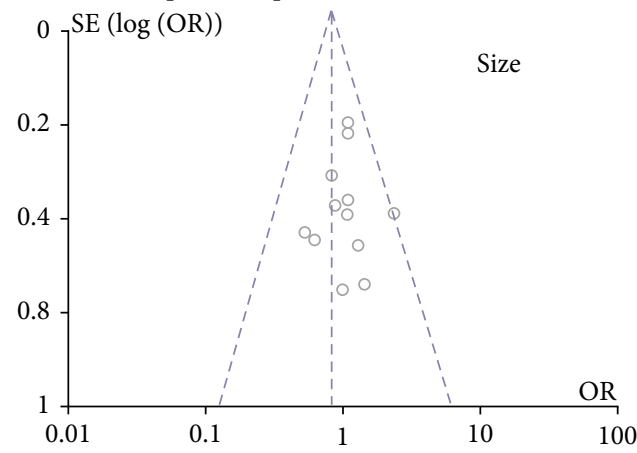

(e)

Funnel plot with pseudo 95\% confidence limits

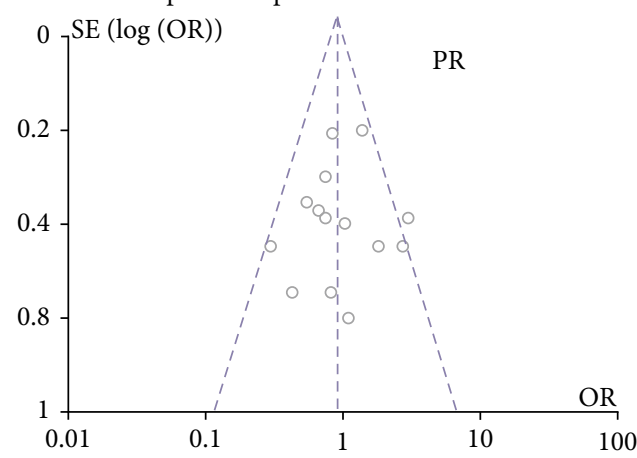

(g)
Funnel plot with pseudo $95 \%$ confidence limits

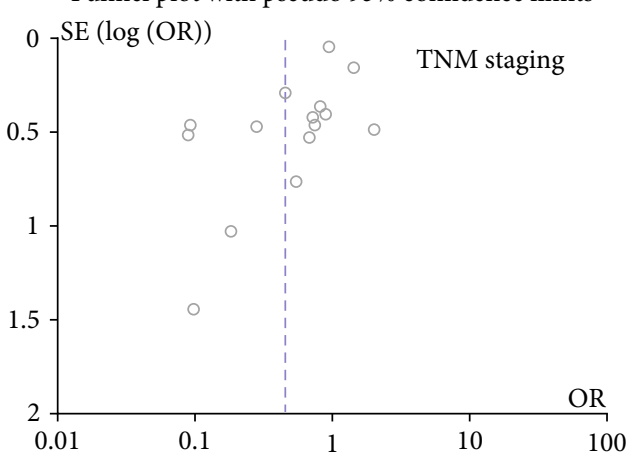

(b)

Funnel plot with pseudo 95\% confidence limits

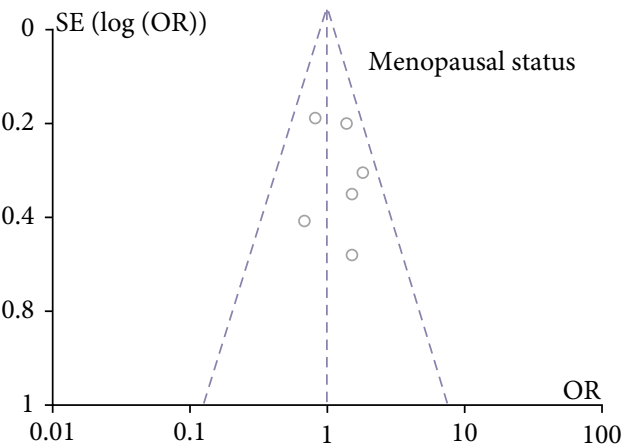

(d)

Funnel plot with pseudo 95\% confidence limits

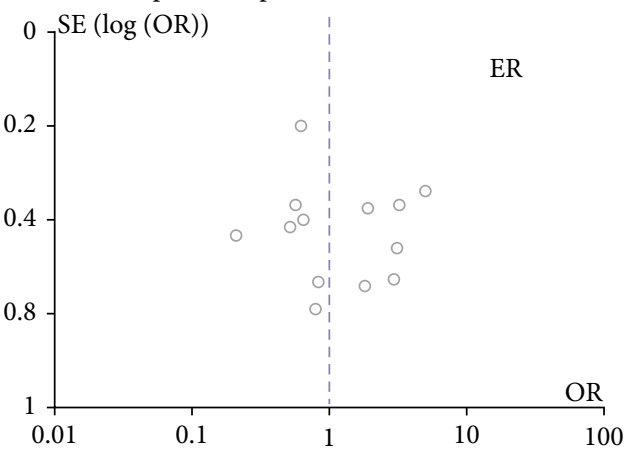

(f)

Funnel plot with pseudo $95 \%$ confidence limits

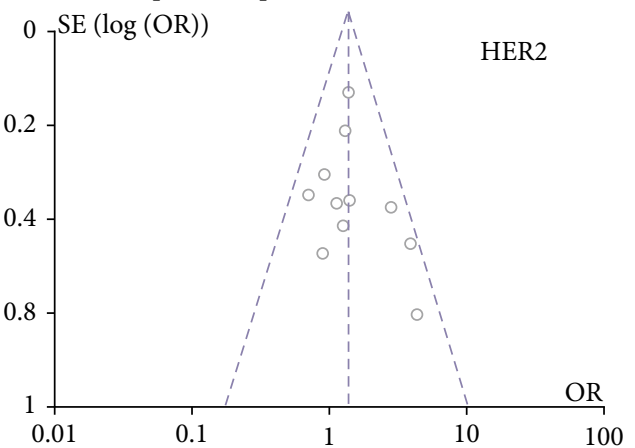

(h)

FIgure 3: Continued. 


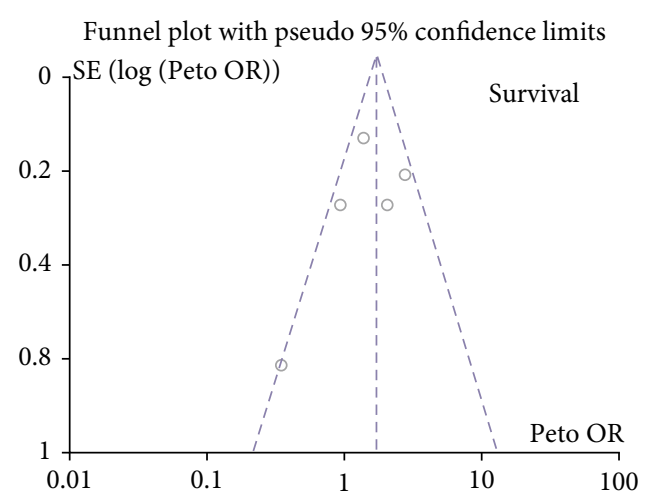

(i)

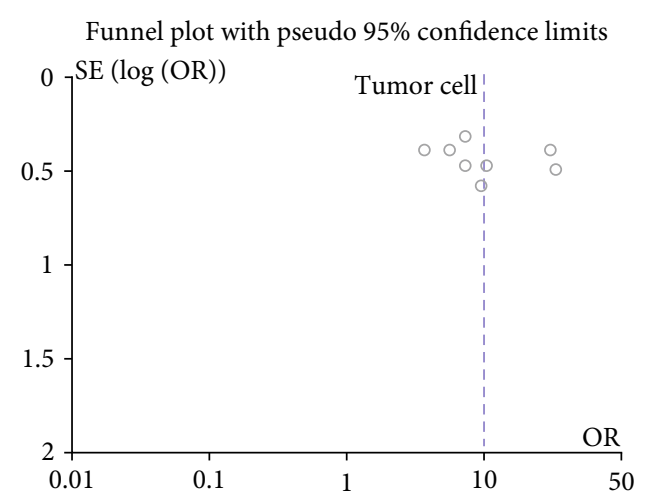

(j)

Funnel plot with pseudo $95 \%$ confidence limits

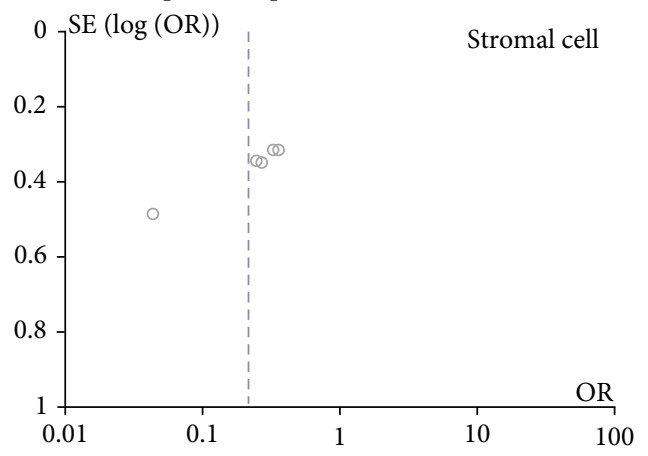

(k)

FIGURE 3: Funnel plot for testing publication bias between SPARC expression and breast cancer. Publication bias was also tested between SPARC expression and clinicopathological features of breast cancer, including (a) histological grade, (b) TNM staging, (c) lymph node metastasis, (d) Menopausal status, (e) size, (f) ER, (g) PR, (h) HER2, and (i) survival. Additionally, publication bias was analyzed based on risk degrees of SPARC expression in (a) tumor cell and (b) stromal cell.

carcinoma and normal tissues. Data on gene expression and the clinical pathology of SPARC were downloaded from the cancer genome atlas (TCGA, http://www.cancer.gov) database using the TCGA-assembler of $\mathrm{R}$ software. The letter code of breast cancer is BRCA. We organized the data and analyzed the mRNA expression of SPARC in breast cancer. At the same time, we analyzed the clinicopathological data and prognosis of tumor patients. Cox risk regression models were used to conduct univariate and multivariate analyses. This model analyzed the effect of risk factors, the hazard ratio (HR), and $95 \%$ confidence interval (CI).

2.4. Statistical Analysis. Revman version 5.3 was used for the data analysis. The results of the comparison between the case group and the control group were expressed as an odds ratio (OR) and $95 \%$ CI. $I^{2}$ statistics were used to determine the heterogeneity between the research results. If a significant level of heterogeneity was found, a fixed-effect model $\left(I^{2}<50 \%, P>0.10\right.$, Mantel-Haenszel method) was used; otherwise, a random effect model $\left(I^{2} \geq 50 \%, P \leq 0.10\right.$, Der Simonian and Laird method) was used. Publication bias was evaluated using a funnel plot, and funnel plot asymmetry was quantified using Begg's test and Egger's test. Cox risk regression models were used for the univariate and multivariate analyses. This model analyzed the effect of risk factors, the hazard ratio, and $95 \%$ CI. A $P$ value of $<0.05$ was consid- ered to indicate a statistically significant difference. All data analyses were conducted using SPSS 19.0 software.

\section{Results}

3.1. Study Selection and Characteristics. As shown in Figure 1 and Table 1, a total of 20 articles that analyzed the relationship between SPARC expression and the clinicopathological characteristics of breast cancer were identified [24-44]. However, only 10 of these articles contained an analysis of normal breast tissues [24, 28-31, 38-42]. Data on the clinicopathological characteristics of breast cancer included histological grade, TNM staging, lymph node metastasis, menopausal status, tumor size, and the presence of ER, PR, and HER2 (Table 2). Finally, only 4 articles were found to include the prognostic features of SPARC expression and its relationship with breast cancer [25, 27, 29, 37].

3.2. Forest Plot of OR for the Association between SPARC Expression and the Clinicopathological Characteristics of Breast Cancer. A total of 10 articles that included data on 571 breast cancer patients and 265 normal controls were found. The expression of SPARC was upregulated in breast cancer tissue compared with normal tissues (Figure 2). Our meta-analysis showed that SPARC expression was associated with TNM staging and histological grade (Table 2). The 

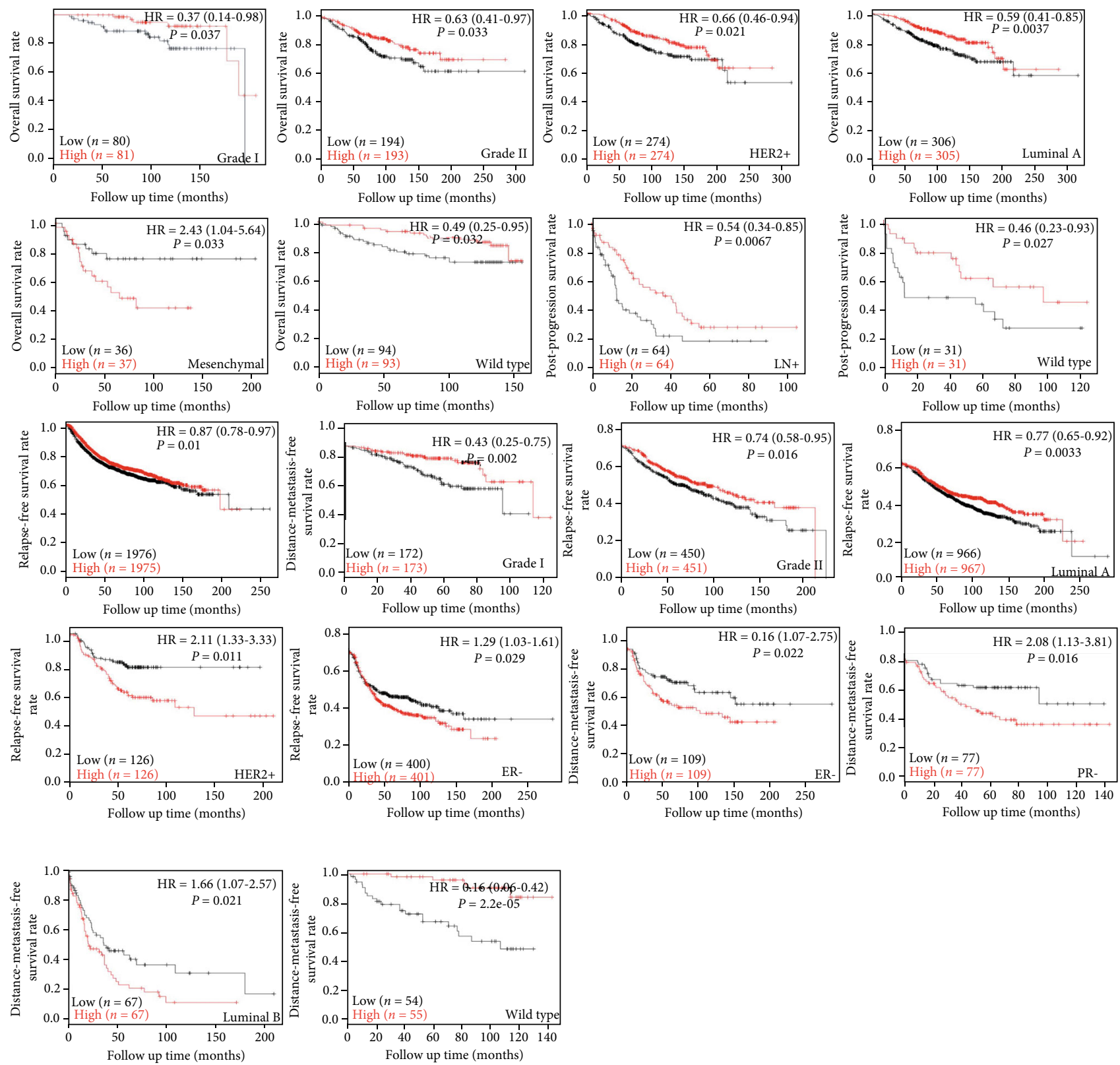

Figure 4: Prognostic value of SPARC mRNA expression in breast cancer patients according to KM-plotter (http://www.kmplot.com).

expression of SPARC was also upregulated in breast stromal cells, compared with tumor cells (Table 2).

3.3. Publication Bias. As shown in Figure 3, the heterogeneity between studies was tested using funnel diagrams. Each study was removed from the pooled analysis to assess the impact of each individual study on the aggregated results using a sensitivity analysis. Egger's test results showed that there was no apparent publication bias in this meta-analysis.

3.4. The Relationship between SPARC Expression and the Bioinformatics Features of Breast Cancer. The KaplanMeier plotter was used to find that lower SPARC expression was negatively correlated with the overall survival rate of grade I/II, Her2+, luminal A, wild type patients, the postpro- gression survival rate of $\mathrm{LN}+$, wild type patients, and the distant metastasis survival rate of wild-type patients (Figure 4, $P<0.05)$. Elevated SPARC expression was positively correlated with the relapse-free survival rate of patients, even when stratified as grade I/II and luminal A patients, but an opposite result was obtained when stratified as Her2+, ER patients. ER, PR, or luminal B patients and elevated SPARC expression produced a shorter distant metastasis survival rate, compared with a lower expression level (Figure $4, P<$ $0.05)$. Based on the TCGA analysis, as well as the databases published by Ma, Radvanyi, Zhao, Curtis, Richardson, Turashvil, Final, and Karnoub, we found that SPARC expression was lower in breast tissues than in invasive or ductal breast carcinoma, invasive lobular, ductal or mixed breast carcinoma, and breast phyllodes tumors. Elevated SPARC 

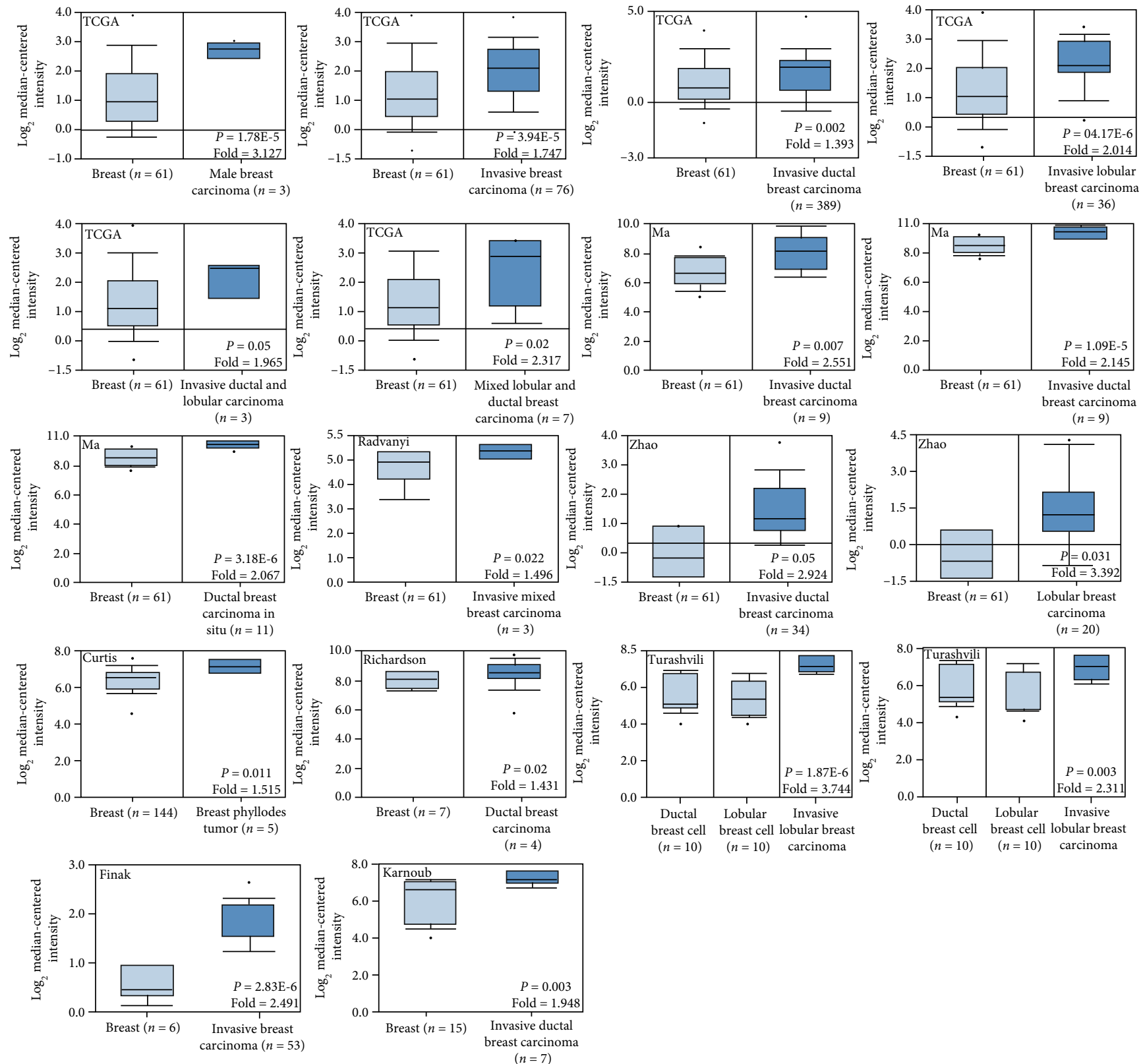

$(n=10) \quad(n=10) \quad$ carcinoma

$(n=10) \quad(n=10) \quad$ carcinom

FIGURE 5: Prognostic value of SPARC mRNA expression in breast cancer patients according to Oncomine database (http://www.oncomine.org).

expression was found in invasive ductal or lobular breast carcinomas, compared with ductal or lobular breast carcinomas (Figure 5, $P<0.05$ ). The Cox univariate analysis of TCGA data showed that age, TNM staging, lymph node metastasis, and distant metastasis were negatively correlated with patient prognosis (Table $3, P<0.05$ ). The Cox multivariate analysis showed that age, TNM staging, lymph node metastasis, and distant metastasis found to be risk factors that for breast cancer patient prognosis (Table $4, P<0.05$ ).

\section{Discussion}

SPARC acts as an antiadhesion protein to regulate growth factors and matrix proteases. SPARC can counteract the effect of $\beta$-FGF in promoting cell proliferation, migration, and mediating angiogenesis, while it can also inhibit $\beta$ FGF receptor autophosphorylation and ERK 1/2 activation [43]. Additionally, SPARC can inhibit VEGF-mediated endothelial cell proliferation and directly bind to PDGF-B and interfere with its binding to fibroblast receptors [44, 45]. TGF- $\beta$ can mediate the production of SPARC proteins. With low levels of TGF- $\beta$, expression levels have been found in the glomerular mesangial cells of SPARC knockout mice [3]. SPARC can mediate the expression of MMP-1 and MMP-9 in peripheral blood monocytes and the expression of MMP-1, MMP-3, and MMP-9 in fibroblasts [46].

Studies have found that SPARC is mainly expressed in mesenchymal cells in gastric cancer tissue. The expression of SPARC was negatively correlated with differentiation, Lauren classification, lymph node metastasis, and clinical 
TABLE 3: Univariate analysis of prognostic risk factors in the patients with breast cancer.

\begin{tabular}{|c|c|c|c|}
\hline Characteristics & Patients (\%) & Relative risk (95\% CI) & $P$ value \\
\hline \multicolumn{4}{|l|}{ Sex } \\
\hline Female & 1065 (98.9) & \multirow{2}{*}{$0.852(0.119-6.102)$} & \multirow{2}{*}{0.873} \\
\hline Male & $12(1.1)$ & & \\
\hline \multicolumn{4}{|l|}{ Age(years) } \\
\hline$<60$ & $599(56.0)$ & \multirow{2}{*}{$0.516(0.371-0.719)$} & \multirow{2}{*}{$<0.001^{*}$} \\
\hline$\geq 60$ & $470(44.0)$ & & \\
\hline \multicolumn{4}{|l|}{ TNM staging } \\
\hline I-II & $792(73.4)$ & \multirow{2}{*}{$0.384(0.272-0.543)$} & \multirow{2}{*}{$<0.001^{*}$} \\
\hline III-IV & $259(24.6)$ & & \\
\hline \multicolumn{4}{|c|}{ Depth of invasion } \\
\hline- & $281(26.2)$ & \multirow{2}{*}{$0.734(0.497-1.084)$} & \multirow{2}{*}{0.120} \\
\hline+ & $792(73.8)$ & & \\
\hline \multicolumn{4}{|c|}{ Lymph node metastasis } \\
\hline- & $504(47.6)$ & \multirow{2}{*}{$0.468(0.352-0.672)$} & \multirow{2}{*}{$<0.001^{*}$} \\
\hline+ & $555(52.4)$ & & \\
\hline \multicolumn{4}{|c|}{ Distant metastasis } \\
\hline- & $893(97.6)$ & \multirow{2}{*}{$0.208(0.124-0.349)$} & \multirow{2}{*}{$<0.001^{*}$} \\
\hline+ & $22(2.4)$ & & \\
\hline
\end{tabular}

CI: confidence interval; TNM: tumor node metastasis.

stage, while lymph node metastasis was an independent prognostic factor for patients with gastric cancer [47-49]. Sato et al. found that SPARC expression at mRNA level in cancer tissues was significantly higher than that of normal adjacent tissues [50]. Previous research has shown that SPARC methylation occurs at a high rate in gastric cancer tissues and that promoter DNA methylation can inhibit SPARC expression in gastric cancer cells [51]. SPARC was also highly expressed in esophageal and liver cancer tissues and was closely related to the degree of malignancy, but its expression was low in pancreatic and colon cancer tissues $[13,15,52,53]$. SPARC expression was significantly lower in ovarian cancer tissues than in normal tissues and in patients with a poorly differentiated and larger omentum [54]. SPARC expression could inhibit the production of interleukin-6 in ovarian cancer tissues and decrease levels of peritoneal metastasis caused by ovarian cancer. SPARC could also block the transformation of vascular endothelial cells from the G1 phase to the S phase and induce the apoptosis and migration of vascular endothelial cells $[55,56]$. Similar results were observed in endometrial cancer but not in cervical cancer $[57,58]$. SPARC expression was also higher in lung squamous cell carcinoma than in lung adenocarcinoma. SPARC was found to be synthesized and secreted by tumor stromal cells [59], and its expression was associated with an acidic environment and the necrosis of tumor tissues, as its expression levels were higher in tumor necrosis areas [60]. The results of multivariate and univariate analyses demonstrated the prognostic value of SPARC expression in determining the overall survival rate [59].

SPARC protein expression was significantly associated with interstitial remodeling, the loss of CD34, and $\alpha$ SMA expression in invasive ductal carcinoma and inter-
TABLE 4: Multivariate analysis of clinicopathological variables for the survival of the patients with breast cancer.

\begin{tabular}{lcc}
\hline Clinicopathological parameters & Relative risk (95\% CI) & $P$ \\
\hline SPARC expression (+) & $0.855(0.597-1.223)$ & 0.390 \\
Age ( $\geq 60$ years) & $2.070(1.425-3.007)$ & $<0.001^{*}$ \\
Sex (female) & $1.753(0.242-12.705)$ & 0.578 \\
Depth of invasion (T2-4) & $1.123(0.709-1.778)$ & 0.622 \\
Lymph node metastasis (+) & $1.622(1.026-2.563)$ & $0.038^{*}$ \\
Distant metastasis (+) & $2.547(1.310-4.950)$ & $0.006^{*}$ \\
TNM staging (III-IV) & $1.707(1.063-2.742)$ & $0.027^{*}$ \\
\hline
\end{tabular}

CI: confidence interval; TNM: tumor node metastasis.

fered with TGF- $\beta 1$ signaling, which allowed it to play a role in tumor progression [38]. SPARC was highly expressed in breast cancer tissues and was associated with TNM staging and lymph node metastasis. The Cox analysis showed that TNM staging and lymph node metastasis were risk factors that affected the prognosis of patients. RT-PCR results showed that SPARC was highly expressed at the mRNA level in breast cancer tissues [61, 62]. Our research results also elevated that high SPARC expression was associated with TNM staging and histological grade. The univariate analysis showed that age, TNM staging, lymph node metastasis, and distant metastasis were associated with a poor prognosis in breast cancer patients. The analysis conducted using the Cox proportional hazard regression model showed that age, TNM staging, lymph node metastasis, and distant metastasis are essential factors that affect the survival time of breast cancer patients. 
SPARC expression at protein and mRNA levels showed opposite trends in breast cancer. The mRNA level expression of a particular gene does not necessarily have a linear relationship with the expression level of its translated product protein due to several reasons. First, gene expression is regulated at various levels, and regulation at the transcription level is only an intermediate link. Second, posttranscriptional translation and posttranslational regulation all contribute to the expression of the final protein. Finally, the mRNA degradation, protein degradation, and modified folding may cause inconsistencies between mRNA and protein expression levels of a given protein.

\section{Conclusion}

SPARC plays a complex role in tumorigenesis and development. At the same time, SPARC expression is upregulated in breast cancer patients. SPARC is positively related with TNM staging and histological grade of breast cancer patients. SPARC expression can be employed as a good marker for the prognosis of patients with cancers, which will provide new methods and ideas for preventive treatment.

\section{Conflicts of Interest}

The authors declare that they have no competing interests.

\section{References}

[1] J. D. Termine, H. K. Kleinman, S. W. Whitson, K. M. Conn, M. L. McGarvey, and G. R. Martin, "Osteonectin, a bonespecific protein linking mineral to collagen," Cell, vol. 26, no. 1, pp. 99-105, 1981.

[2] A. Chlenski and S. L. Cohn, "Modulation of matrix remodeling by SPARC in neoplastic progression," Seminars in Cell \& Developmental Biology, vol. 21, no. 1, pp. 55-65, 2010.

[3] K. Brune, S. M. Hong, A. Li et al., "Genetic and epigenetic alterations of familial pancreatic cancers," Cancer Epidemiology and Prevention Biomarkers, vol. 17, no. 12, pp. 35363542, 2008.

[4] W. J. Wang and C. L. Mei, "Research progress of SPARC," Chinese Journal of Clinical Medicine, vol. 5, pp. 65-68, 2004.

[5] M. W. M. Schellings, D. Vanhoutte, M. Swinnen et al., "Absence of SPARC results in increased cardiac rupture and dysfunction after acute myocardial infarction," Journal of Experimental Medicine, vol. 206, no. 1, pp. 113-123, 2009.

[6] O. Gaillard, P. Lebon, and J. Delattre, "Bidirectional signaling between the cytoskeleton and integrins," Current Opinion in Cell Biology, vol. 11, no. 2, pp. 274-286, 1999.

[7] Q. Yan and E. H. Sage, "SPARC a matricellular glycoprotein with important biological functions," Journal of Histochemistry \& Cytochemistry, vol. 47, no. 12, pp. 1495-1505, 1999.

[8] A. D. Bradshaw, "Diverse biological functions of the SPARC family of proteins," International Journal of Biochemistry and Cell Biology, vol. 44, no. 3, pp. 480-488, 2012.

[9] E. W. Raines, T. F. Lane, M. L. Iruela-Arispe, R. Ross, and E. H. Sage, "The extracellular glycoprotein SPARC interacts with platelet-derived growth factor (PDGF)-AB and $-\mathrm{BB}$ and inhibits the binding of PDGF to its receptors," Proceedings of the National Academy of Sciences, vol. 89, no. 4, pp. 12811285, 1992.
[10] C. Lau, R. Poon, S. Cheung, W. C. Yu, and S. T. Fan, "SPARC and Hevin expression correlate with tumour angiogenesis in hepatocellular carcinoma," Journal of Pathology, vol. 210, no. 4, pp. 459-468, 2006.

[11] G. P. Nagaraju and B. F. EI-Rayes, "SPARC and DNA methylation: possible diagnostic and therapeutic implications in gastrointestinal cancers," Cancer Letters, vol. 328, no. 1, pp. 1017, 2013.

[12] A. Gao, X. Zuo, Q. G. Liu, X. Lu, W. Guo, and L. Tian, "Methylation of PARP-1 promoter involved in the regulation of benzene-induced decrease of PARP-1 mRNA expression," Toxicology Letters, vol. 195, no. 2-3, pp. 114-118, 2010.

[13] N. Sato, N. Fukushima, N. Maehara et al., "SPARC/osteonectin is a frequent target for aberrant methylation in pancreatic adenocarcinoma and a mediator of tumor-stromal interactions," Oncogene, vol. 22, no. 32, pp. 5021-5030, 2003.

[14] J. Nie, B. Chang, D. O. Traktuev et al., "IFATS collection: combinatorial peptides identify $\alpha 5 \beta 1$ integrin as a receptor for the matricellular protein SPARC on adipose stromal cells," Stem Cells, vol. 26, no. 10, pp. 2735-2745, 2008.

[15] E. Yang, H. J. Kang, K. H. Koh, H. Rhee, N. K. Kim, and H. Kim, "Frequent inactivation of SPARC by promoter hypermethylation in colon cancers," International Journal of Cancer, vol. 121, no. 3, pp. 567-575, 2007.

[16] M. J. Tang and I. T. Tai, "A novel interaction between procaspase 8 and SPARC enhances apoptosis and potentiates chemotherapy sensitivity in colorectal cancers," Journal of Biological Chemistry, vol. 282, no. 47, pp. 34457-34467, 2007.

[17] S. Cheetham, M. J. Tang, F. Mesak, H. Kennecke, D. Owen, and I. T. Tai, "SPARC promoter hypermethylation in colorectal cancers can be reversed by 5 -Aza- $2^{\prime}$ deoxycytidine to increase SPARC expression and improve therapy response," British Journal of Cancer, vol. 98, no. 11, pp. 1810-1819, 2008.

[18] F. Prada, L. G. Benedetti, A. I. Bravo, M. J. Alvarez, C. Carbone, and O. L. Podhajcer, "SPARC endogenous level, rather than fibroblast-produced SPARC or stroma reorganization induced by SPARC, is responsible for melanoma cell growth," Journal of Investigative Dermatology, vol. 127, no. 11, pp. 2618-2628, 2007.

[19] D. Massi, A. Franchi, L. Borgognoni, U. M. Reali, and M. Santucci, "Osteonectin expression correlates with clinical outcome in thin cutaneous malignant melanomas," Human Pathology, vol. 30, no. 3, pp. 339-344, 1999.

[20] C. Schultz, N. Lemke, and S. Ge, "Secreted protein acidic and rich in cysteine promotes glioma invasion and delays tumor growth in vivo," Cancer Research, vol. 62, pp. 6270-6277, 2002.

[21] S. A. Rempel, S. G. Ge, and J. A. Gutierrez, "SPARC: a potential diagnostic marker of invasive meningiomas," Clinical Cancer Research, vol. 5, no. 2, pp. 237-241, 1999.

[22] A. Nishie, K. Masuda, M. Otsubo et al., "High expression of the Cap 43 gene in infiltrating macrophages of human renal cell carcinomas," Clinical cancer research, vol. 7, no. 7, pp. 21452151, 2001.

[23] R. Thomas, L. D. True, J. A. Bassuk, P. H. Lange, and R. L. Vessella, "Differential expression of osteonectin/SPARC during human prostate cancer progression. Clinical cancer research an official journal of the american association for cancer research," vol. 6, no. 3, pp. 1140-1149, 2000.

[24] K. Cui and B. Y. Li, "The relationship between the expression level of SPARC and the occurrence, development, invasion 
and metastasis of breast cancer," Journal of Hunan Normal University, vol. 14, pp. 57-60, 2017.

[25] X. D. Xu, L. Xu, Y. J. Li, Z. H. Li, Z. Li, and Y. E. Teng, "Expression and clinical significance of SPARC protein in breast cancer," Journal of China Medical University, vol. 43, pp. 493-498, 2014.

[26] Q. F. Zhou, S. L. Yue, S. Q. Qi, Z. Z. Zhao, Y. Su, and Y. Lin, "Effects of secreted acidic protein and androgen receptor rich in cysteine on the prognosis of patients with triple negative breast cancer," Modern Practical Medicine, vol. 32, pp. 180$182,2020$.

[27] F. Chen, L. Zhu, H. Chen, S. Z. Chen, and Y. H. Xu, "Clinical significance of secreted protein acidic and rich in cysteine expression in breast cancer tissue," The Chinese Journal of Clinical Pharmacology, vol. 33, pp. 18-20, 2017.

[28] C. Yan and W. Hong, "Observation of SPARC protein expression in breast cancer," Shandong Medical Journal, vol. 56, pp. 63-64, 2016.

[29] Y. Chen, S. Z. Liu, and P. Zhang, "The expression of SPARC and Notch-1 in breast cancer and their clinical significanceS," Journal of Hubei University for Nationalities.Medical Edition, vol. 35, pp. 14-18, 2018.

[30] L. L. Gao, G. H. Yu, and J. M. Zhang, "Expression and significance of SIRT1 and SPARC in breast invasive ductal carcinoma tissues," China Medical Herald, vol. 12, pp. 16-18, 2015.

[31] X. M. Zhang, J. W. Chen, H. H. Hu, G. Z. Lu, and Z. R. Yang, "Expression of SPARC in invasive ductal carcinoma of breast," Journal of Shanghai Jiaotong University, vol. 28, pp. 14451448, 2008.

[32] J. L. Lindner, S. Loibl, C. Denkert et al., "Expression of secreted protein acidic and rich in cysteine (SPARC) in breast cancer and response to neoadjuvant chemotherapy ${ }^{\dagger}, "$ Annals of Oncology, vol. 26, no. 1, pp. 95-100, 2015.

[33] J. J. Ma, S. Gao, X. J. Xie et al., "SPARC inhibits breast cancer bone metastasis and may be a clinical therapeutic target," Oncology Letters, vol. 14, no. 5, pp. 5876-5882, 2017.

[34] A. J. Zhu, P. Yuan, F. Du, R. X. Hong, X. Y. Ding, and X. Q. Shi, "SPARC overexpression in primary tumors correlates with disease recurrence and overall survival in patients with triple negative breast cancer," Oncotarget, vol. 7, no. 47, pp. 7662876634, 2016.

[35] W. Guo, M. Zhang, Y. Chen, and S. Y. Gao, "The clinical significance of secreted protein acidic and rich in cysteine expression in breast cancer tissue and its association with prognosis," Journal of Cancer Research \& Therapeutics, vol. 13, no. 5, pp. 833-836, 2017.

[36] A. K. Witkiewicz, B. Freydin, I. Chervoneva et al., "Stromal CD10 and SPARC expression in ductal carcinoma in situ (DCIS) patients predicts disease recurrence," Cancer Biology \& Therapy, vol. 10, no. 4, pp. 391-396, 2010.

[37] G. Watkins, A. Douglas-Jones, R. Bryce, R. E. Mansel, and W. G. Jiang, "Increased levels of SPARC (osteonectin) in human breast cancer tissues and its association with clinical outcomes," Prostaglandins leukotrienes \& essential fatty acids, vol. 72, no. 4, pp. 267-272, 2005.

[38] P. J. Barth, R. Moll, and A. Ramaswamy, "Stromal remodeling and SPARC (secreted protein acid rich in cysteine) expression in invasive ductal carcinomas of the breast," Virchows Archiv, vol. 446, no. 5, pp. 532-536, 2005.

[39] X. Y. Sun, G. H. Yu, and J. M. Zhang, "Correlation between SPARC and MMP-2 expression in breast cancer tissues," Chi- nese Journal of Cancer Prevention and Treatment, vol. 22, pp. 674-677, 2015.

[40] X. M. Liu, G. H. Yu, J. M. Zhang, S. Z. Liu, Y. F. Gao, and F. Su, "Expression and significance of SPARC and VEGF in breast invasive ductal carcinoma tissues," Chinese Journal of Cancer Prevention and Treatment, vol. 19, pp. 1061-1065, 2012.

[41] S. Q. Zhang and W. Ma, "Expression of cysteine-rich secreted protein in breast cancer and its clinical significance," Maternal and Child Health Care of China, vol. 31, pp. 5157-5159, 2016.

[42] X. Y. Sun, G. H. Yu, and J. M. Zhang, "SPARC and Her-2's expression in breast cancer tissue," China Medicine and Pharmacy, vol. 4, pp. 18-21, 2014.

[43] M. J. Socha, N. Said, Y. Dai et al., "Aberrant promoter methylation of_Sparc_in ovarian cancer," Neoplasia, vol. 11, no. 2, p. 126-IN1, 2009.

[44] N. Said, M. J. Socha, J. J. Olearczyk, A. A. Elmarakby, J. D. Imig, and K. Motamed, "Normalization of the ovarian cancer microenvironment by SPARC," Molecular Cancer Research, vol. 5, no. 10, pp. 1015-1030, 2007.

[45] T. S. Mantoni, R. R. E. Schendel, F. Rödel et al., "Stromal SPARC expression and patient survival after chemoradiation for non-resectable pancreatic adenocarcinoma," Cancer Biology \& Therapy, vol. 7, no. 11, pp. 1806-1815, 2008.

[46] S. Arnold, E. Mira, S. Muneer et al., "Forced expression of MMP9 rescues the loss of angiogenesis and abrogates metastasis of pancreatic tumors triggered by the absence of host SPARC," Experimental Biology \& Medicine, vol. 233, no. 7, pp. 860-873, 2008.

[47] Y. Li, J. F. Wu, and W. D. Zhao, "Expression and significance of SPARC and MMP-9 in gastric cancer," Shandong Medical Journal, vol. 55, pp. 48-50, 2015.

[48] M. X. Dong, Y. H. Yang, and J. J. Yu, "Expression of SPARC in gastric cancer tissues and its significance," QILU YIXUE ZAZHI, vol. 26, pp. 105-110, 2011.

[49] M. L. Yang, L. L. Zhai, and L. H. Ma, "Relationship between expression of SPARC and VEGF with tumor angiogenesis in gastric carcinoma," Chinese Journal of Diagnostic Patholog, vol. 19, pp. 52-55, 2012.

[50] T. Sato, T. Oshima, N. Yamamoto et al., "Clinical significance of SPARC gene expression in patients with gastric cancer," Journal of Surgical Oncology, vol. 108, no. 6, pp. 364-368, 2013.

[51] Z. Y. Chen, J. L. Zhang, H. X. Yao et al., “Aberrant methylation of the _SPARC_ gene promoter and its clinical implication in gastric cancer," Scientific Reports, vol. 4, no. 1, p. 7035, 2015.

[52] H. Porte, J. P. Triboulet, L. Kotelevets et al., "Overexpression of stromelysin-3, BM-40/SPARC, and MET genes in human esophageal carcinoma: implications for prognosis," Clinical Cancer Research, vol. 4, no. 6, pp. 1375-1382, 1998.

[53] B. B. Le, S. Faouzi, and L. Boussarie, "Osteonectin/SPARC is overexpressed in human hepatocellular carcinoma," The Journal of Pathology, vol. 189, no. 1, pp. 46-52, 1999.

[54] L. L. Yan, Z. M. Cui, and Y. Zheng, "Expression and significance of SPARC in epithelial ovarian tumors," Shandong Medical Journal, vol. 48, pp. 35-37, 2008.

[55] N. A. Said, I. Najwer, M. J. Socha, D. J. Fulton, S. C. Mok, and K. Motamed, "SPARC inhibits LPA-mediated mesothelial-ovarian cancer cell crosstalk," Neoplasia, vol. 9, no. 1, pp. 23-35, 2007.

[56] A. Chlenski, S. Q. Liu, and S. E. Crawford, "SPARC is a key Schwannian-derived inhibitor controlling neuroblastoma tumor angiogenesis," Cancer Research, vol. 62, pp. 73577363, 2003. 
[57] T. Deng and Q. Zhou, "Expression of SPARC in the squamous cervical carcinoma and its clinic significance," Journal of Chongqing Medical University, vol. 36, pp. 60-64, 2011.

[58] L. Wang, Z. M. Cui, and Y. Zheng, "Expression and significance of the SPARC protein in endometrial carcinoma," Progess in modern biomedicine, vol. 11, pp. 905-908, 2011.

[59] Y. Huang, J. Zhang, Y.-Y. Zhao et al., "SPARC expression and prognostic value in non-small cell lung cancer," Chinese Journal of Cancer, vol. 31, no. 11, pp. 541-548, 2012.

[60] M. I. Koukourakis, A. Giatromanolaki, R. A. Brekken et al., "Enhanced expression of SPARC/osteonectin in the tumorassociated stroma of non-small cell lung cancer is correlated with markers of hypoxia/acidity and with poor prognosis of patients," Cancer Research, vol. 63, no. 17, pp. 5376-5380, 2003.

[61] Y. Chen, S. Z. Liu, and P. Zhang, "Expressions of SPARC and Notch-1 in breast cancer and their clinical significance," Journal of Hubei University for Nationalities, vol. 35, pp. 17-21, 2018.

[62] Z. Q. Che, H. Wang, and J. Qi, "Expression of SPARC and clinical significance in breast cancer," The Practical Journal of Cancer, vol. 20, pp. 120-122, 2005. 